criteria of the European Working Group on Sarcopenia in Older People (3) using appendicular lean mass assessed by bioelectric impedance analysis and grip strength - stratifying both by body mass index. We also used the 2016 formula by Scafoglieri et.al to calculate appendicular lean mass (4). This approach is different from previous studies because we combined the new formula with established cut-off values, which incorporate a functional parameter. Muscle function was assessed by hand grip, knee extension strength and peak expiratory flow. Impairment and quality of life were evaluated using the Scleroderma Health Assessment Questionnaire (SHAQ) and Short-Form 36 Health Survey $\left(\mathrm{SF}-36^{\circledR}\right)$. Clinical data were assessed according to standardised procedures.

Results: The prevalence of sarcopenia in our study group was $27.1 \%$.There were no significant differences between patients with and without sarcopenia regarding age $(p=0.838)$ and disease duration $(p=0.832)$. There were significant differences regarding grip strength $(p<0.001)$, knee extension strength $(p=0.003)$, peak flow $(p=0.042), S F-36^{\circledR}$ Physical Function $(p=0.009)$ and the number of immunosuppressive $(p=0.048)$ and other drugs taken $(p=0.037)$. See Table 1.

Table 1 Comparing analysis between two patient groups (Sarcopenia/ No Sarcopenia)

\begin{tabular}{|c|c|c|c|}
\hline Variable & Sarcopenia, $n=35$ & No Sarcopenia, $n=94$ & $\mathbf{p}$ \\
\hline Women & $32(91.4)$ & $85(90.4)$ & 0.862 \\
\hline Age (years) & $59.5 \pm 14.8$ & $59.0 \pm 13.5$ & 0.838 \\
\hline Disease duration (years) & $8.6 \pm 6.0$ & $8.9 \pm 6.9$ & 0.832 \\
\hline Body Mass Index $\left(\mathrm{kg} / \mathrm{m}^{2}\right)$ & $20.6 \pm 2.7$ & $25.5 \pm 5.2$ & $<0.001$ \\
\hline Hand grip strength $(\mathrm{kgF})$ & $10.3 \pm 6.1$ & $18.7 \pm 8.0$ & $<0.001$ \\
\hline Knee extension strength (kgF) & $13.6 \pm 7.2$ & $22.3 \pm 16.3$ & 0.003 \\
\hline Peak flow (1/min) & $305.8 \pm 102.0$ & $348.3 \pm 101.9$ & 0.042 \\
\hline SF- $36^{\circ}$ Physical Health SUM & $40.5 \pm 18.1$ & $48.5 \pm 19.6$ & 0.043 \\
\hline SF- $36^{\circ}$ Physical Function & $41.7 \pm 27.1$ & $54.3 \pm 27.4$ & 0.026 \\
\hline SHAQ score & $0.66 \pm 0.12$ & $0.64 \pm 0.07$ & 0.010 \\
\hline IPAQ (level of activity-high) & $5(14.3)$ & $28(29.8)$ & 0.073 \\
\hline Number of medication & $6.2 \pm 3.8$ & $4.8 \pm 3.2$ & 0.037 \\
\hline $\begin{array}{l}\text { Number of immunosuppressive } \\
\text { medication }\end{array}$ & $1.4 \pm 1.1$ & $1.0 \pm 1.0$ & 0.048 \\
\hline
\end{tabular}

Conclusions: There is a high prevalence of sarcopenia in patients with SSc even among younger patients. Sarcopenic patients have a significant increase in physical impairment and a decreased quality of life regarding their physical health. Taking a higher number of immunosuppressive or other drugs was identified as a risk factor for sarcopenia. This could be due to severe organ involvement being a confounder or an indication for the immunological component in the pathophysiology of secondary sarcopenia. This suggests that sarcopenia might be linked to severe disease or multimorbiditiy in SSc patients.

References:

[1] Wang C, Bai L. Sarcopenia in the elderly: basic and clinical issues. Geriatrics \& gerontology international. 2012 Jul;12(3):388-96.

[2] Dogan SC, Hizmetli S, Hayta E et.al. Sarcopenia in women with rheumatoid arthritis. European journal of rheumatology. 2015 Jun;2(2):57-61.

[3] Cruz-Jentoft AJ, Baeyens JP, Bauer JM et.al. Sarcopenia: European consensus on definition and diagnosis: Report of the European Working Group on Sarcopenia in Older People. Age and ageing. 2010 Jul;39(4):412-23.

[4] Scafoglieri A, Clarys JP, Bauer JM et.al. Predicting appendicular lean and fat mass with bioelectrical impedance analysis in older adults with physical function decline - The PROVIDE study. Clinical nutrition (Edinburgh, Scotland). 2016 Apr 28.

Disclosure of Interest: None declared

DOI: 10.1136/annrheumdis-2017-eular.5532

\section{SAT0334 CORRELATING MULIPLEX PLASMA CYTOKINE ANALYSIS WITH RIGHT HEART CATHETERISATION FINDINGS IN SCLERODERMA ASSOCIATED PULMONARY ARTERIAL HYPERTENSION}

C.H. Ho ${ }^{1}$, S. Nihtyanova ${ }^{2}$, B. Ahmed $\mathrm{Abdi}^{2}$, G. Coghlan ${ }^{3}$, C. Denton ${ }^{2}$, B. Schreiber ${ }^{4}$, V.H. Ong ${ }^{2} .{ }^{1}$ Department of Medicine, Queen Elizabeth Hospital, Kowloon, Hong Kong; ${ }^{2}$ Centre for Rheumatology and Connective Tissue Diseases, University College London Medical School, Royal Free Hospital; ${ }^{3}$ Department of Cardiology; ${ }^{4}$ Department of Rheumatology, Royal Free Hospital, London, United Kingdom

Background: Right heart catheterisation $(\mathrm{RHC})$ is an important test for the diagnosis and subclassification of pulmonary hypertension $(\mathrm{PH})$, one of the serious manifestations of systemic sclerosis (SSc). It provides haemodynamic information for diagnosis and management. Routine RHC in SSc cases with suspected $\mathrm{PH}$ permits blood sampling to explore plasma levels of key cytokines that may be markers of mediators of pulmonary arterial hypertension (PAH) pathogenesis.

Objectives: This is an exploratory study to assess for associations between plasma cytokine levels and haemodynamic assessment from RHC in SSc patients. Methods: SSc patients undergoing routine RHC were recruited. Indications for referral included suspected $\mathrm{PH}$, or those on treatment for established $\mathrm{PH}$ for assessment of response to therapy. Blood samples were collected during RHC in consented patients. Demographic and clinical data were obtained. Haemodynamic data from RHC including mean right atrial pressure (mRAP), mean pulmonary arterial pressure (mPAP), pulmonary capillary wedge pressure (PCWP), pulmonary vascular resistance (PVR), cardiac output (CO) and cardiac index $(\mathrm{Cl})$ were recorded. Plasma samples were analysed using a bead-based multiplex platform for IL1b, IL4, IL6, IL10, IL17A, IL17F, IL21, IL22, IL23, IL25, IL31, IL33, IFNg, sCD40L and TNFa (Bio-Rad Pro Assays). Plasma from a small group of healthy controls were also analysed for comparison. For analytes found to be below the lower limit of detection of the assays, they were assumed to be equal to the lower limit of the detection as per the assay's protocol.

Results: 32 SSc patients were recruited. Their mean age was 59.4-year-old, and 31 of them $(97 \%)$ were female. Most $(n=29,91 \%)$ had limited cutaneous SSc. The commonest antibody was anti-centromere antibody ( $n=16,55 \%)$, followed by anti-U3RNP antibody $(n=4,14 \%)$. None of these patients had significant pulmonary fibrosis ( $\geq 20 \%$ involvement on HRCT thorax scan). PAH, defined as mPAP $\geq 25 \mathrm{mmHg} \& \mathrm{PCWP} \leq 15 \mathrm{mmHg}$, was diagnosed at RHC in 26 patients $(81 \%)$.

Among the proteins tested, only the level of SCD40L was significantly different among the three groups, namely SSc with $\mathrm{PH}$, SSc without $\mathrm{PH}$ and healthy controls $(p=0.008)$. SCD40L was also the only biomarker which was significant higher among patients with SSc (with and without $\mathrm{PH}$ ) than healthy controls $(\mathrm{p}=0.0284)$.

Interestingly, there was a weak negative correlation between SCD40L and mPAP (correlation coefficient $=-0.35, \mathrm{p}=0.0486$ ). There was a moderately strong positive correlation between mRAP and IL4 (correlation coefficient $=0.57, \mathrm{p}=0.0006$ ) and IL10 (correlation coefficient $=0.51, p=0.0028$ ). We found no evidence for association between any of the biomarkers and cardiac output, cardiac index, PCWP or PVR

Conclusions: Our data suggest that some individual plasma proteins may correlate with specific RHC haemodynamic parameters. Future studies will extend these findings and explore whether combining multiple analytes may give stronger non-invasive prediction of relevant haemodynamic variables and could be used in detection or monitoring of PAH in SSc.

References:

[1] Proc Natl Acad Sci USA. 2015 May19;122(20):E2677-86.

[2] Ann Am Thorac Soc. 2016 Jan;13(1)25-30.

Disclosure of Interest: None declared

DOI: 10.1136/annrheumdis-2017-eular.5798

\section{SAT0335 SERUM KYNURENINE/TRYPTOPHAN (KYN/TRP) RATIO AND NEOPTERIN (NEO) LEVELS ARE RAISED IN SYSTEMIC SCLEROSIS (SSC) AND ASSOCIATE WITH SPECIFIC CLINICAL AND AETIO-PATHOGENETIC FEATURES}

C. Campochiaro ${ }^{1}$, S.D. Lytton ${ }^{2}$, B. Ahmed Abdi ${ }^{1}$, V.H. Ong ${ }^{1}$, C.P. Denton ${ }^{1}$. ${ }^{1}$ Centre for Rheumatology and Connective Tissue Diseases, Royal Free Hospital and UCL Division of Medicine, London, United Kingdom; ${ }^{2}$ SeraDiaLogistics Benediktenwandstr 7, 81545, München, Germany

Background: The enzymes IDO degrades Trp into Kyn and is induced by IFN-gamma/IL-1. Neo is produced by monocytes/macrophages after IFN-gamma stimulation through the enzyme GTPCH and is a biomarker for monitoring immune activation in several diseases [1]. The activity of GTPCH is induced in parallel to IDO and the Kyn/Trp ratio has been suggested to be a direct measure of IDO activity [2].

Objectives: To assess serum level of Kyn, Trp, Neo and Kyn/Trp ratio in SSc and potential associations with specific disease features.

Methods: 60 SSc pts and 10 healthy controls $(\mathrm{HC})$ were recruited and serum levels of Kyn, Trp and Neo were measured. Kyn/Trp ratio was calculated. The results were then correlated with specific disease features: disease duration, limited or diffuse disease, autoantibody profile (anti-RNA pol III (ARA), antitopoisomerase (ATA) and anti-centromere (ACA)), inflammatory markers, $\mathrm{Hb}$ level, concurrent modified Rodnan skin score (mRSS), peak mRSS, pulmonary fibrosis (PF), pulmonary arterial hypertension (PAH), history of scleroderma renal crisis (SRC), GI involvement, vasculopathy, environmental exposure, oncology history, smoking status, immunosuppressive treatment, NT-proBNP and urate levels. Non-parametric statistical tests were used.

Results: Kyn/Trp ratio was higher in SSc compared to HC (mean 49.97 \pm 32.77 $[41.50-58.43]$ vs $22.5 \pm 6.3[13.5-32.5] \mu \mathrm{mol} / \mathrm{mmol}, p<0.05)$ and, more specifically, dcSSc showed higher ratio compared to IcSSc that had higher ratio than $\mathrm{HC}$ (mean $56.58 \pm 39.62$ [43.18 - 69.99] vs 40.05 \pm 13.93 [34.16 $45.93]$ vs $22.5 \pm 6.3[17.99-27.01] \mu \mathrm{mol} / \mathrm{mmol}$ respectively, $p<0.05)$. Moreover, 